\title{
The Effects of a Self-Adapted, Jaw Repositioning Mouthpiece and Jaw Clenching on Muscle Activity during Vertical Jump and Isometric Clean Pull Performance
}

\author{
Charles Allen (Corresponding author) \\ Exercise Science Program, Florida Southern College, 111 Lake Hollingsworth Drive, Lakeland, FL 33801, USA \\ E-mail: callen@flsouthern.edu \\ Yang-Chieh Fu
}

Department of Health, Exercise Science and Recreation Management, University of Mississippi, P.O. Box 1848, University, Mississippi 38677, USA E-mail: ycfu@olemiss.edu

John C. Garner

Department of Kinesiology and Health Promotion, Troy University, 600 University Avenue, Troy, Alabama 36082, USA

E-mail: jcgarner@troy.edu

Received: 28-05- 2016

doi:10.7575/aiac.ijkss.v.4n.3p.42
Accepted: 12-07- 2016

Published: 31-07- 2016

URL: http://dx.doi.org/10.7575/aiac.ijkss.v.4n.3p.42

\begin{abstract}
Purpose: The purpose of this study was to investigate the effects of a self-adapted, jaw repositioning mouthpiece and jaw clenching on muscle activity during the countermovement vertical jump (CMVJ) and isometric mid-thigh clean pull (MTCP). Methods: Thirty-six healthy, recreationally trained males ( $\mathrm{n}=36$; age, $23 \pm 2.8$ years; height, $178.54 \pm 9.0 \mathrm{~cm}$; body mass, $83.09 \pm 7.8 \mathrm{~kg}$ ) completed maximal CMVJ and MTCP assessments under six experimental conditions: jaw repositioning mouthpiece plus clenching $(\mathrm{MP}+\mathrm{C})$, jaw repositioning mouthpiece with jaw relaxed $(\mathrm{MP})$, traditional mouthguard plus clenching $(\mathrm{MG}+\mathrm{C})$, traditional mouthguard with jaw relaxed $(\mathrm{MG})$, no mouthpiece plus clenching $(\mathrm{NoMP}+\mathrm{C})$ and no mouthpiece with jaw relaxed (NoMP) while muscle activity of the dominant leg medial gastrocnemius $(\mathrm{G})$, medial hamstring $(\mathrm{H})$, vastus medialis (VMO), and erector spinae (ES) was recorded. Results: Repeated measures ANOVA revealed no changes in MTCP muscle activation for any mouthpiece or clench condition. Jaw clenching, regardless of mouthpiece condition, significantly improved prime mover muscle activation during CMVJ $(p<.001)$. Prime mover muscle activation was significantly greater during CMVJ assessment for jaw repositioning mouthpiece and no mouthpiece conditions over the use of a traditional mouthguard $(p<.001)$, but the repositioning mouthpiece did not lead to improved muscle activation compared to no mouthpiece $(p>.05)$. Conclusion: These findings support jaw clenching as a viable technique to elicit concurrent activation potentiation (CAP) of prime mover muscle activity during dynamic but not isometric physical activity.
\end{abstract}

Keywords: jaw repositioning mouthpiece, jaw clenching, concurrent activation potentiation, muscle activation

\section{Introduction}

The use of jaw-aligning mouthpieces to alleviate the symptoms associated with temporomandibular joint disorder (TMD) is quite common and has been purported to improve various aspects of performance (Smith, 1978; Kaufman, 1980; Kaufman and Kaufman, 1984). Recently, the popularity of jaw-aligning devices for the purposes of performance enhancement has grown. These mouthguards, which in addition to the jaw repositioning properties, provide protection to the orofacial structures similar to traditional protective mouthguards. They also come in mouthpiece form, which changes the mandibular-maxillary relationship without the protective properties. Dental impressions taken by a dental practitioner are used to custom fit the jaw repositioning devices to the individual, which leads to an expensive end product; however, there are self-adaptable boil-and-bite versions available. These products can be designed to fit the upper or lower jaw, depending on the specific oral appliance model and individual preference, and have small acrylic bite plates that inhibit direct contact of the upper and lower molar teeth when the mouth is closed. This changes the temporomandibular joint relationship, pulling the mandible down and slightly forward, which mimics the jaw position achieved with the TMD treatment devices.

Several studies have examined these devices and their effects on a variety of physiological variables with mixed results (Garner \& McDivitt, 2009; Garner \& Miskimin, 2009; Garner et al, 2011a; Garner et al, 2011b). A positive relationship between a customized, jaw repositioning mouthpiece and aerobic endurance exercise performance has been reported with changes in airway openings (Garner \& McDivitt, 2009) and several parameters of respiratory exchange including $\mathrm{VO}_{2}, \mathrm{VO}_{2} / \mathrm{kg}$, and $\mathrm{VCO}_{2}$ (Garner et al, 2011a). Another investigation reported significant improvements in auditory 
reaction times when the same customized repositioning mouthpiece was worn compared to no mouthpiece use (Garner \& Miskimin, 2009). Stress hormone response following a vigorous bout of resistance exercise was significantly attenuated when a customized, jaw repositioning mouthpiece was implemented in comparison to no mouthpiece conditions (Garner et al, 2011b). Researchers examined salivary cortisol levels at various time points during and postresistance training exercise. While cortisol levels were similar for the duration of the exercise bout for both the mouthpiece and no mouthpiece conditions, cortisol levels at 10 minutes post exercise was significantly lower when the mouthpiece was used, suggesting a direct relationship between jaw repositioning mouthpiece use and post-exercise attenuation of cortisol.

Studies examining the effects of jaw repositioning devices on various measures of force production have also been conducted (Arent et al, 2010; Dunn-Lewis et al, 2012; Allen et al, 2014). No improvement in vertical jump performance variables or one-repetition maximum bench press performance was reported with the use of a self-adapted, jaw repositioning mouthpiece compared to no mouthpiece (Allen et al, 2014). Similarly, the effects of two jaw repositioning mouthguards on strength, power and a myriad of other assessments were investigated with no improvements in any performance measure compared to placebo mouthguard and no mouthguard (Golem \& Arent, 2015). Conversely, significant improvements in vertical jump height and peak power during the 30 second Wingate Anaerobic Power Test were reported when a neuromuscular dentistry-based, customized mouthguard was used by professional and collegiate athletes compared to tests without a mouthguard in the same participant group (Arent et al, 2010). Dunn-Lewis et al, (2012) examined a self-adapted, jaw repositioning mouthguard's effects on a myriad of performance variables in highly trained males and females. Power and force production during the bench throw test were significantly greater in both sexes under the mouthpiece condition. Additionally, significant improvements were reported for the mouthpiece condition in males only for force production and power during the plyo-press power quotient assessment and rate of power production during the vertical jump assessment. The use of a customized, jaw repositioning mouthguard was also reported to enhance the concurrent activation potentiation (CAP) effects of jaw clenching by promoting a more aligned and forceful contraction of the masticatory muscles (Busca et al, 2016). Grip strength performance, which was greater when the jaw was clenched than when the jaw was relaxed, improved to a greater extent when participants clenched the jaw while wearing the customized jaw repositioning mouthguard.

Proposed mechanisms underlying jaw repositioning mouthguard and mouthpiece use are varied depending upon the performance outcome of interest. Early practitioners of neuromuscular dentistry proposed improved proprioceptive function (Jakush, 1982). Another proposed mechanism of interest involves improved neuromuscular response due to proper jaw alignment (Garner et al, 2011b). Increased genioglossus muscle contraction, demonstrated to lead to a relaxation of the pharyngeal airway, was proposed as one explanation for improved gas exchange parameters during treadmill running when a performance mouthpiece was worn compared to no mouthpiece condition (Garner et al, 2011b). These authors also reported increased electromyography activity of the genioglossus muscle when the jaw repositioning mouthpiece was worn (Garner et al, 2011b). An increase in neuromuscular activity may explain, at least in part, the previously reported improvements in muscle force production. However, the effects of jaw repositioning mouthpiece use on prime mover muscle activation during physical activity have not yet been reported in the literature. Consequently, this investigation sought to examine the effects of a self-adapted, jaw-repositioning mouthpiece on muscle activation during power and force production activities. Additionally, the effects of maximal jaw clenching while wearing the jaw-repositioning mouthpiece were also examined as jaw clenching has been shown to impact force production and muscle activation during physical activity (Ebben et al, 2010b). It was hypothesized that jaw clenching during the selected assessments would lead to improvements in muscle activation over non-clenched conditions and the addition of the jaw repositioning mouthpiece might further improve muscle activation.

\section{Methods}

This study examined how jaw clenching and jaw alignment via a self-adapted, jaw repositioning mouthpiece impacted muscle activation during maximum countermovement vertical jump (CMVJ) and maximum isometric mid-thigh clean pull (MTCP) assessments. A within-subjects design was used in which participants repeated the assessments under each experimental condition.

\subsection{Subjects}

Thirty six $(n=36)$ physically active and recreationally resistance trained males, aged 18-30 years, completed the research protocol. Participants were considered physically active if they engaged in routine resistance training exercise for a minimum of three days per week for the previous month. None of the participants ( $\mathrm{n}=36$; age, $23 \pm 2.8$ years; height, $178.54 \pm 9.0 \mathrm{~cm}$; body mass, $83.09 \pm 7.8 \mathrm{~kg}$ ) reported current or past history of TMD, and all were free of physical injury and illness at the time of testing. All participants signed the University approved Institutional Review Board consent documents.

\subsection{Experimental Controls}

To ensure no dietary abnormalities throughout testing, a dietary journal documenting all food and beverage intake for the 72 hours prior to the initial testing session was required. Additionally, 24-hour dietary recalls were also reported for both remaining testing days. Participants were also asked to refrain from any non-prescription supplementation/drug use throughout the study with caffeine being the only exception. Participants were asked to maintain normal use or nonuse of caffeine for the duration of the study. To ensure adequate hydration status for assessment, consumption of 5-7 milliliters of water per kilogram of body weight four hours prior to each testing session was prescribed (American 
Dietetic Association, 2009). A urine sample was provided by all participants on each testing day which was analyzed for specific gravity via dipstick (BTNX Inc; Markham, Ontario, Canada) to ensure euhydration status prior to testing. Participants maintained their normal exercise routines, however, they were asked to refrain from exercise 24 hours prior to a testing session. Finally, participants were asked to maintain their normal sleeping patterns as best as possible throughout their study participation. Dietary records were analyzed by a registered dietitian to ensure individual consistency throughout study participation, and questioning by the primary investigator was conducted each testing day prior to the onset of assessment to determine participant adherence to exercise, nutritional, and sleep requests.

\subsection{Procedures}

Experimental testing consisted of four laboratory visits. The initial visit involved participant prescreening, obtaining informed consent, basic anthropometric measurements, provision of mouthpieces, and familiarization with all testing procedures. The three remaining laboratory visits were data collection sessions lasting approximately one hour and were separated by approximately one week. All testing times were scheduled within one hour of the time of day of the previous testing session to account for diurnal variation. There were three oral appliance conditions which consisted of a self-adapted, jaw repositioning mouthpiece (ArmourBite Mouthpiece; Under Armour, Baltimore, MD, USA), a traditional mouthguard (Cramer Mouth Guard; Cramer Products Inc, Gardner, KS, USA), and no mouthpiece. To account for jaw clenching during assessment, two jaw musculature conditions, jaw clenched and jaw relaxed, were included for a total of six experimental conditions which were as follows: jaw repositioning mouthpiece plus clenching $(\mathrm{MP}+\mathrm{C})$, jaw repositioning mouthpiece with jaw relaxed (MP), traditional mouthguard plus clenching $(\mathrm{MG}+\mathrm{C})$, traditional mouthguard with jaw relaxed (MG), no mouthpiece plus clenching (NoMP+C) and no mouthpiece with jaw relaxed (NoMP). The experimental conditions were randomized for all participants. Both jaw clenched and jaw relaxed trials for each respective mouthpiece condition were performed within a testing session separated by a twenty minute washout period to allow for recovery. To control for clenching during the jaw relaxed trials, the participants were instructed to breathe through pursed lips which is consistent with previously published research (Ebben et al, 2008b; Ebben et al, 2010a).

\subsubsection{Maximum Voluntary Contraction Assessment}

During each testing visit, the maximum CMVJ and isometric MTCP trials were preceded by maximum voluntary contraction (MVC) assessment. Following dynamic warm up and prior to each assessment of CMVJ and MTCP, participants were asked to perform three MVCs for each of the selected musculature. Participants maximally contracted the selected muscles for three seconds, and EMG activity was collected for five seconds including one second prior to and immediately following those contractions. These MVC were analyzed for peak signals, and were used to determine percent activation of the selected musculature during performance of the assessments.

\subsubsection{Countermovement Jump Assessment}

CMVJ assessment procedures via a Vertec ${ }^{\circledR}$ device (Sports Imports, Columbus, OH, USA) were consistent with previously described methods (McGuigan, 2016). The participants were instructed to determine their maximum reach height by standing flat-footed, directly underneath the Vertec device, reaching up with the dominant hand to push forward the highest vane that could be reached. The height of the device was then increased to accommodate a maximal effort CMVJ. The participant was then instructed to perform each CMVJ trial without moving the feet prior to take off, to jump maximally, and to tap the highest vane possible at the apex of the jump. Trials were recorded as the vertical distance, to the nearest one-half inch between the reach height and height of vane tapped during the jump. Each participant was permitted three trials separated by 30 seconds for each testing condition. The trial producing the highest jump was used for analysis.

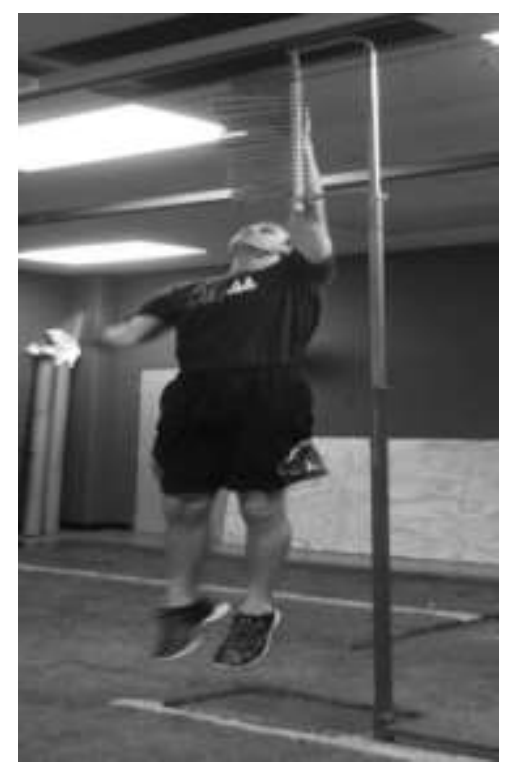

Image 1. Countermovement Vertical Jump Assessment

Description: Participant reaches up with the dominant hand at the apex of the jump to tap the highest vane possible. 


\subsubsection{Isometric Mid-Thigh Clean Pull Assessment}

Procedures for MTCP assessment were consistent with those previously reported (Kawamori, 2006). A Jones machine (BodyCraft, Inc., Sunbury, OH, USA) was used to facilitate MTCP assessment. The machine was modified so that the bar was fixed and unmovable. A goniometer was used to standardize hip and knee angles to flexed positions of $125^{\circ}$ and $140^{\circ}$ respectively, with as little variance between participants as the Jones machine adjustments would allow. The participants used a double overhand, closed grip in which the thumb was wrapped around the bar. Additionally, nylon weightlifting straps were used to remove hand size and grip strength as potentially limiting factors. When instructed, the participant exerted maximal force onto the floor while pulling against the fixed barbell for three seconds. Thirty seconds rest was provided between trials to ensure recovery. Three trials were afforded to each participant. The trials yielding the best performance were utilized for further analysis.

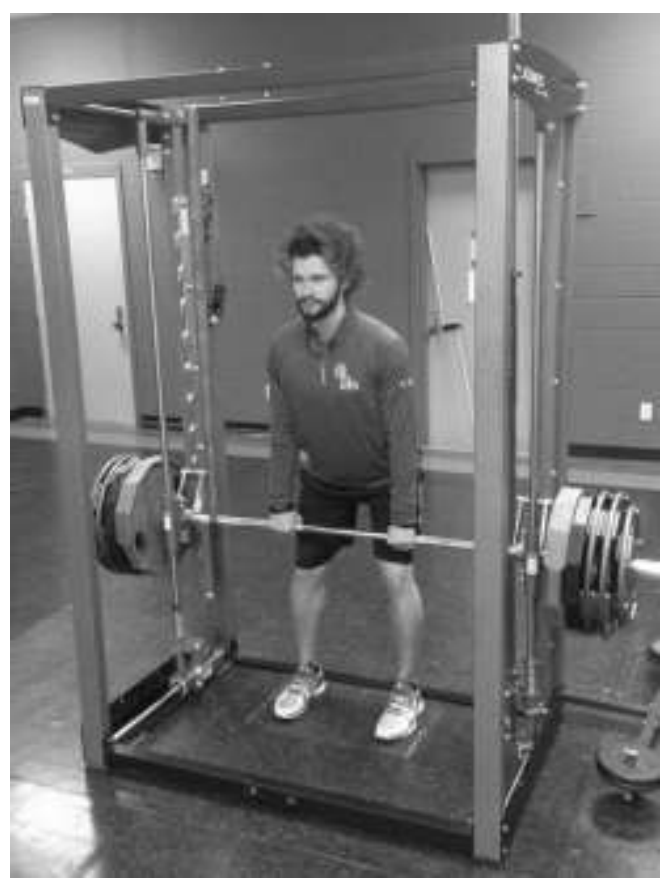

Image 2. Mid-Thigh Clean Pull Assessment

Description: The barbell is affixed by nylon straps and weight added to the barbell to prevent movement of the Jones machine.

\subsection{Electromyography}

Bipolar surface electromyography (EMG) was recorded at $1000 \mathrm{~Hz}$ during CMVJ and MTCP assessment on the participants' dominant side during all three laboratory testing visits. Electrodes (EME Company, Baton Rouge, LA, USA) $5 \mathrm{~cm}$ in length and $3 \mathrm{~cm}$ in width were placed $3 \mathrm{~cm}$ apart, as measured from the electrode center, at each location with a ground electrode on the tibial head. Skin preparation for all electrodes included shaving of the hair and abrasion of the skin around electrode site followed by cleansing with an alcohol swab. Data was recorded from the medial head of the gastrocnemius $(\mathrm{G})$, medial hamstring $(\mathrm{H})$, oblique fibers of the vastus medialis (VMO), and erector spinae (ES). Specific electrode placement followed the recommendations found on the Surface Electromyography for the NonInvasive Assessment of Muscles website (The SENAM Project). EMG data collection was facilitated using an 8channel electromyography system (Noraxon USA Inc., Scottsdale, AZ, USA) and raw EMG data were processed with a $4^{\text {th }}$ order Butterworth bandpass filter $(10-300 \mathrm{~Hz})$ via MatLab software (The MathWorks, Inc., Natick, MA, USA).

\subsection{Statistical Analyses}

A 3 x 2 (mouthpiece $\mathrm{x}$ clench condition) repeated measures ANOVA was conducted to analyze each of the dependent variables for interaction and main effect significance. In cases where conditions of sphericity were not met, the Greenhouse-Geisser correction estimate was used if $\varepsilon<.75$, and the Huynh-Feldt correction estimate was used if $\varepsilon>.75$. Pair-wise comparisons utilized a Bonferroni confidence interval adjustment. Paired sample t-tests were utilized to determine specific differences when interactions between mouthpiece and clench condition were observed. All analyses were performed with an a priori alpha level of $p \leq 0.05$. Sample size was also determined a priori using G*Power 3.1 software (Faul et al, 2009). Data were analyzed using IBM Statistics package software, version 22.0 (IBM SPSS Statistics, Armonk, NY, USA).

\section{Results}

For all CMVJ and MTCP EMG measures, data are expressed as a percentage of activation relative to MVC EMG signal. For MVC EMG, data represents the peak EMG signal recorded during the trial with greatest muscle activity. 


\subsection{MVC Data}

Peak EMG signal for all four muscles assessed are presented in Table 1 below. There was main effect significance for jaw clenching $(p=0.019)$ for gastrocnemius muscle activity only. There was mouthpiece*clench interaction significance for both the gastrocnemius and erector spinae peak EMG signal. Further analysis revealed that the peak gastrocnemius EMG signal was significantly greater for the MG+C compared to MG condition, and peak erector EMG signal was significantly greater for the NoMP compared to the NoMP+C condition.

Table 1. Peak EMG signal during MVC trials (mV)

\begin{tabular}{lllllll}
\hline & MP & MP+C & MG & MG+C & NoMP & NoMP+C \\
\hline $\mathrm{G}$ & $.570 \pm .22$ & $.562 \pm .24$ & $.498 \pm .21$ & $* .604 \pm .25$ & $.544 \pm .24$ & $.589 \pm .27$ \\
\hline $\mathrm{H}$ & $.514 \pm .30$ & $.518 \pm .25$ & $.525 \pm .29$ & $.546 \pm .30$ & $.515 \pm .31$ & $.497 \pm .29$ \\
\hline $\mathrm{VMO}$ & $.349 \pm .19$ & $.347 \pm .19$ & $.307 \pm .16$ & $.334 \pm .18$ & $.304 \pm .17$ & $.328 \pm .18$ \\
\hline $\mathrm{ES}$ & $.239 \pm .08$ & $.241 \pm .08$ & $.260 \pm .12$ & $.262 \pm .12$ & $* .290 \pm .15$ & $.244 \pm .11$ \\
\hline
\end{tabular}

Description: Data are expressed as mean \pm standard deviation. An asterisk $(*)$ indicates a significant difference $(\mathrm{p}<$ 0.05 ) between clench conditions. $\mathrm{G}=$ medial gastrocnemius; $\mathrm{H}=$ medial hamstring; $\mathrm{VMO}=$ vastus medialis obliquus; $\mathrm{ES}=$ erector spinae; $\mathrm{MP}=\mathrm{jaw}$ repositioning mouthpiece; $\mathrm{MP}+\mathrm{C}=\mathrm{jaw}$ repositioning mouthpiece and jaw clenching; $\mathrm{MG}=$ protective mouthguard; $\mathrm{MG}+\mathrm{C}=$ protective mouthguard and jaw clenching; $\mathrm{NoMP}=$ no mouthpiece; $\mathrm{NoMP}+\mathrm{C}=\mathrm{no}$ mouthpiece, clenching only.

\subsection{CMVJ EMG Data}

There was significant main effects for mouthpiece and clench conditions for the G, H, and VMO but not ES muscle activity. Data illustrating these findings are found in Tables 2 and 3 respectively. A significant mouthpiece*clench interaction was also observed for muscle activity of all four muscles of interest. Post-hoc analysis of this interaction revealed that the $\mathrm{MG}+\mathrm{C}$ condition elicited significantly greater percentages of muscle activation than the $\mathrm{MG}$ condition for all four muscles.

Table 2. Percentage of muscle activation during CMVJ relative to MVC

\begin{tabular}{llll}
\hline & MP & MG & NoMP \\
\hline $\mathrm{G}$ & $76.88 \pm 3.18$ & $* 48.44 \pm 3.55$ & $74.55 \pm 3.83$ \\
\hline $\mathrm{H}$ & $90.82 \pm 7.13$ & $* 76.32 \pm 6.48$ & $96.21 \pm 9.01$ \\
\hline $\mathrm{VMO}$ & $246.99 \pm 19.34$ & $* 153.54 \pm 12.11$ & $233.19 \pm 21.46$ \\
\hline $\mathrm{ES}$ & $146.61 \pm 13.07$ & $150.297 \pm 13.22$ & $155.86 \pm 23.50$ \\
\hline
\end{tabular}

Description: Data are expressed as mean \pm standard error. An asterisk $(*)$ indicates a significant difference $(\mathrm{p}<0.05)$ relative to the other mouthpiece conditions. $\mathrm{G}=$ medial gastrocnemius; $\mathrm{H}=$ medial hamstring; $\mathrm{VMO}=\mathrm{vastus}$ medialis obliquus; $\mathrm{ES}=$ =erector spinae; $\mathrm{MP}=\mathrm{jaw}$ repositioning mouthpiece; $\mathrm{MG}=$ protective mouthguard; NoMP=no mouthpiece.

Table 3. Percentage of muscle activation during CMVJ relative to MVC

\begin{tabular}{llll}
\hline & Jaw Clenched & Jaw Relaxed & $p$ Value \\
\hline $\mathrm{G}$ & $76.36 \pm 3.09^{*}$ & $56.89 \pm 2.64$ & $p<0.001$ \\
\hline $\mathrm{H}$ & $97.27 \pm 6.42^{*}$ & $78.29 \pm 6.61$ & $p=0.001$ \\
\hline $\mathrm{VMO}$ & $236.48 \pm 16.31^{*}$ & $186.00 \pm 14.29$ & $p<0.001$ \\
\hline $\mathrm{ES}$ & $156.20 \pm 13.34$ & $145.65 \pm 13.73$ & $p=0.327$ \\
\hline
\end{tabular}

Description: Data are expressed as mean \pm standard error. An asterisk $(*)$ indicates a significant difference $(\mathrm{p}<0.05)$ between clench conditions. $\mathrm{G}=$ medial gastrocnemius; $\mathrm{H}=$ medial hamstring; $\mathrm{VMO}=$ vastus medialis obliquus; $\mathrm{ES}=$ erector spinae.

\subsection{MTCP EMG Data}

MTCP EMG data are represented in Tables 4 and 5. There were no significant interaction or main effects for percent activation of any muscle for any treatment condition.

Table 4. Percentage of muscle activation during MTCP relative to MVC

\begin{tabular}{llll}
\hline & MP & MG & NoMP \\
\hline $\mathrm{G}$ & $20.14 \pm 2.09$ & $20.67 \pm 2.08$ & $19.57 \pm 1.96$ \\
\hline $\mathrm{H}$ & $42.20 \pm 4.11$ & $47.55 \pm 6.51$ & $49.48 \pm 5.17$ \\
\hline $\mathrm{VMO}$ & $73.39 \pm 6.83$ & $73.46 \pm 7.05$ & $76.32 \pm 8.18$ \\
\hline ES & $125.54 \pm 7.66$ & $126.81 \pm 7.86$ & $124.27 \pm 8.86$ \\
\hline
\end{tabular}


Description: Data are expressed as mean \pm standard error. $\mathrm{G}=$ medial gastrocnemius; $\mathrm{H}=$ medial hamstring; $\mathrm{VMO}=$ vastus medialis obliquus; $\mathrm{ES}=$ erector spinae; $\mathrm{MP}=$ jaw repositioning mouthpiece; $\mathrm{MG}=$ =protective mouthguard; NoMP=no mouthpiece.

Table 5. Percentage of muscle activation during MTCP relative to MVC

\begin{tabular}{llll}
\hline & Jaw Clenched & Jaw Relaxed & $p$ Value \\
\hline $\mathrm{G}$ & $20.45 \pm 1.77$ & $19.80 \pm 2.01$ & $p=0.574$ \\
\hline $\mathrm{H}$ & $48.97 \pm 5.47$ & $43.84 \pm 3.93$ & $p=0.217$ \\
\hline $\mathrm{VMO}$ & $74.23 \pm 6.17$ & $74.55 \pm 7.27$ & $p=0.927$ \\
\hline ES & $126.20 \pm 6.74$ & $124.88 \pm 7.75$ & $p=0.764$ \\
\hline
\end{tabular}

Description: Data are expressed as mean \pm standard error. $\mathrm{G}=$ medial gastrocnemius; $\mathrm{H}=$ medial hamstring; $\mathrm{VMO}=$ vastus medialis obliquus; $\mathrm{ES}=$ erector spinae.

\section{Discussion}

The aim of this investigation was to determine changes in muscle activity due to wearing a self-adapted, jaw repositioning mouthpiece during maximum CMVJ and isometric MTCP assessment. Additionally, due to jaw clenching potentially impacting the results, the authors sought to determine whether the observed changes could be attributed exclusively to jaw clenching, jaw alignment by the use of a repositioning mouthpiece, or if the presence of both conditions led to synergistic results.

\subsection{Mouthpiece Conditions}

There was no difference in percent activation between MP and NoMP conditions during CMVJ and MTCP performance for any of the four muscles examined. While performance enhancements such as improved respiratory exchange parameters (Garner et al, 2011a), increased vertical jump height and anaerobic power (Arent et al, 2010), and improved force production variables (Dunn-Lewis et al, 2012) have been attributed to the use of jaw repositioning appliances, increased relative muscle activation does not appear to be among them. One possible explanation for this may be related to the jaw repositioning mouthpiece design. Many of the previous investigations demonstrating performance improvements as the result of jaw repositioning appliance use employed customized versions fabricated by dental practitioners specifically for the individual user (Arent et al, 2010; Garner \& McDivitt, 2009; Garner \& Miskimin, 2009; Garner et al, 2011a; Garner et al, 2011b) . The current study utilized a self-adapted, jaw repositioning mouthpiece which utilizes a typical boil-and-bite fitting procedure. It is possible that a customized jaw repositioning appliance may prove beneficial in augmenting muscle activation during forceful exertion.

Interestingly, percent activation for the $\mathrm{G}, \mathrm{H}$, and $\mathrm{VMO}$ was significantly lower during the MG condition compared to the MP and NoMP conditions during the CMVJ assessment. This is an important finding considering the recommended and requisite usage of similar mouthguards during sports such as lacrosse and American football (ADA, 2006; NCAA, 2011). Although important in providing safety and protection of the teeth and mouth from potential injury during competition, many athletes have negative perceptions of mouthguards due to breathing and verbal communication difficulties (Ferrari et al, 2002). Additionally, some athletes suspect that mouthguards have detrimental effects on performance (Bourdin et al, 2006; Gardiner et al, 2000). Upon the completion of the current investigation, participants were polled regarding their preference of the oral appliance conditions in the study. The responses reflect similar discontent with the MG used compared to the MP and NoMP conditions. Thirty one of 36 participants indicated a preference for either MP or NoMP conditions over the MG condition, with the most common reason given being discomfort in the MG condition. Of the five participants who indicated a preference for the MG condition, all cited familiarity from previous participation in sports requiring such mouthguards as the reason for their preference. It is possible that the MG condition, being unfamiliar and uncomfortable to the majority of the participants, created an awkward and distracting performance environment, leading to the observed detriment in muscle activation.

\subsection{Jaw Clenching Conditions}

Clenching the jaw elicited significantly greater percent activation of the G, H, and VMO but not the ES, compared to the non-clench condition and regardless of mouthpiece condition during CMVJ performance. Erector spinae activity was not different between clench conditions. Ebben (2006) introduced and defined concurrent activation potentiation (CAP) as the ergogenic advantage of increased prime mover performance as the result of simultaneous remote voluntary contraction (RVC) such as clenching the jaw, and touted CAP as the reason for improved force production variables during various physical activities (Ebben et al, 2008a; Ebben et al, 2008b; Ebben et al, 2010a; Ebben et al, 2010b). Considering this definition, the current findings are logical. For the CMVJ, the G, H, and VMO would be considered prime movers. The ES, although active during the CMVJ, would not be considered a prime mover for this activity, and as such, would not be potentiated during CMVJ performance. A previous study investigating muscle activity during isokinetic knee extension and flexion revealed significantly higher muscle activity for prime mover musculature when RVC including jaw clenching were utilized (Ebben et al, 2010b). Muscle activity of the movement antagonist as well as homologous contralateral musculature was not changed. These findings, as well as the findings of the current investigation, support the specificity of CAP to the prime movers involved in the activity of interest. 
In contrast, jaw clenching failed to lead to a significant change in muscle activity in comparison to the non-clench condition for any muscle examined during performance of the isometric MTCP. These findings are consistent with previous research as well (Garceau et al, 2012). Muscle activity during isometric knee extension with the incorporation of jaw clenching as well as other RVC was no different than isometric knee extension without RVC (Garceau et al, 2012). Although muscle activity was not significantly different between RVC and no RVC conditions, PF and RFD were significantly improved under RVC conditions (Garceau et al, 2012). Increased neural drive as a result of functional cortical connections and motor overflow has been proposed as the primary mechanism underlying CAP (Ebben, 2006; Ebben et al, 2008a; Ebben et al, 2008b; Ebben et al, 2010a; Ebben et al, 2010b). The findings of the current study, coupled with those reported by Garceau et al, (2012), suggest that any observed CAP performance improvement during isometric activity would not be due to increased neural drive but other mechanisms not yet known. As stated previously, this investigation sought to discern whether jaw clenching or jaw repositioning mouthpiece use was exclusively responsible for any observed changes in muscle activity and was not designed to determine specific mechanisms leading to those changes. Future research should attempt to reveal those mechanisms. It is important to note that while the current results do not support motor overflow as the underlying mechanism of CAP during isometric activity, it does not negate it either.

Previous research has demonstrated that aggregate RVC elicited CAP to a greater extent than isolated RVC (Ebben et al, 2008b). During isometric knee extension, mean and peak torque values were significantly improved when a single RVC was utilized, however, conditions that combined multiple RVC led to knee extensor torque values greater than the single RVC condition (Ebben et al, 2008b). The current study was concerned specifically with jaw clenching as a variable impacting muscle activity and not with maximizing CAP via the incorporation of multiple RVC. As such, other examples of RVC, such as the Valsalva maneuver, were not incorporated. All participants in the current investigation were given instructions to breathe as normally as possible during the performance assessments, in an attempt to control for the potential CAP effects of holding the breath. It is possible that, with the incorporation of additional RVC, CAP may have been stimulated to a greater degree, and increased muscle activity to the level of statistical significance.

\section{Conclusions}

This is the first study to determine whether observed improvements in muscle activation can be attributed exclusively to jaw repositioning mouthpiece use, jaw clenching, or if both conditions are necessary to achieve ergogenic effects. Jaw clenching, regardless of mouthpiece condition, improved muscle activation during countermovement vertical jump (CMVJ) compared to non-clench conditions. Although muscle activation was greater during CMVJ assessment for jaw repositioning mouthpiece and no mouthpiece conditions over the use of a traditional mouthguard, the repositioning mouthpiece did not lead to improved muscle activation compared to no mouthpiece. No changes were observed in isometric mid-thigh clean pull (MTCP) muscle activation for any mouthpiece or clench condition. These findings support jaw clenching as a viable technique to elicit concurrent activation potentiation (CAP) of prime mover muscle activity during dynamic but not isometric physical activity. Future studies should examine customized jaw repositioning mouthguards or mouthpieces and their effects on muscle activation.

\section{References}

ADA council on access, prevention and inter-professional relations; ADA council on scientific affairs. (2006). Using mouthguards to reduce the incidence and severity of sports-related oral injuries. The Journal of the American Dental Association, 137, 1712-1720.

Allen, CR, Dabbs, NC, Zachary, CS, Garner, JC. (2014). The acute effect of a commercial bite-aligning mouthpiece on strength and power in recreationally trained men. Journal of Strength and Conditioning Research, 28(2), 499-503.

American Dietetic Association. (2009). Position of the American Dietetic Association, Dietitians of Canada, and the American College of Sports Medicine: Nutrition and Athletic Performance. The Journal of the American Dental Association, 109, 509-527.

Arent, SM, McKenna, J, Golem, DL. (2010). Effects of a neuromuscular dentistry-designed mouthguard on muscular endurance and anaerobic power. Comparative Exercise Physiology, 7, 73-79.

Bourdin, M, Brunet-Patru, I, Hager, PE, Allard, Y, Hager, JP, Lacour, JR, Moyen, B. (2006). Influence of maxillary mouthguards on physiological parameters. Medicine \& Science in Sports \& Exercise, 38(8), 1500-1504.

Busca, B, Morales, J, Solana-Tramunt, M, Miro, A, and Garcia, M. (2016). Effects of jaw clenching while wearing a customized bite-aligning mouthpiece on strength in healthy young men. Journal of Strength and Conditioning Research, 30(4), 1102-1110.

Dunn-Lewis, C, Luk, H, Comstock, BA, Szivak, TK, Hooper, DR, Kupchak, BR, Watts, AM, Putney, BJ, Hydren, JR, Volek, JS, Denegar, CR, Kraemer, WJ. (2012). The effects of a customized over-the-counter mouth guard on neuromuscular force and power production in trained men and women. Journal of Strength and Conditioning Research, 26, 1085-1093.

Ebben, WP. (2006). A brief review of concurrent activation potentiation: theoretical and practical constructs. Journal of Strength and Conditioning Research, 20, 985-991. 
Ebben, WP, Flanagan, EP, Jensen, RL. (2008). Jaw clenching results in concurrent activation potentiation during the countermovement jump. Journal of Strength and Conditioning Research, 22, 1850-1854.

Ebben, WP, Kaufmann, CE, Fauth, ML, Petushek EJ. (2010). Kinetic analysis of concurrent activation potentiation during back squats and jump squats. Journal of Strength and Conditioning Research, 24(6), 1515-1519.

Ebben, WP, Leigh, DH, Geiser, CF. (2008). The effect of remote voluntary contractions on knee extensor torque. Medicine \& Science in Sports \& Exercise, 40(10), 1805-1809.

Ebben, WP, Petushek, EJ, Fauth, ML, Garceau, LR. (2010). EMG analysis of concurrent activation potentiation. Medicine \& Science in Sports \& Exercise, 42(3), 556-562.

Faul, F, Erdfelder, E, Buchner, A, Lang, AG. (2009). Statistical power analyses using G*Power 3.1: Tests for correlation and regression analyses. Behavior Research Methods, 40(4), 1149-1160.

Ferrari, CH, Ferreria de Mederios, JM. (2002). Dental trauma and level of information: mouthguard use in different contact sports. Dental Traumatology, 18, 144-147.

Garceau, LR, Petushek, EJ, Fauth, ML, Ebben, WP. (2012). Effect of remote voluntary contractions on isometric prime mover torque and electromyography. Journal of Exercise Physiology Online, 15(4), 40-46.

Gardiner, DM, Ranalli, DN. (2000). Attitudinal factors influencing mouthguard utilization. Dental Clinics of North America, 44, 53-65.

Garner, DP, Dudgeon, WD, McDivitt, EJ. (2011). The effects of mouthpiece use on cortisol levels during an intense bout of resistance exercise. Journal of Strength and Conditioning Research, 25, 2866-2871.

Garner, DP, Dudgeon, WD, Scheett, TP, McDivitt, EJ. (2011). The effects of mouthpiece use on gas exchange parameters during steady-state exercise in college-aged men and women. The Journal of the American Dental Association, 142, 1041-1047.

Garner, DP, McDivitt, EJ. (2009). Effects of mouthpiece use on airway openings and lactate levels in healthy college males. Compendium of Continuing Education in Dentistry, 30, 9-13.

Garner, DP, Miskimin, J. (2009). Effects of mouthpiece use on auditory and visual reaction time in college males and females. Compendium of Continuing Education in Dentistry, 30, 14-17.

Golem, DL, Arent, SM. (2016). Effects of over-the-counter jaw-repositioning mouth guards on dynamic balance, flexibility, agility, strength, and power in college-aged male athletes. Journal of Strength and Conditioning Research, 29, 500-512.

Jakush J. (1982). Divergent views: Can dental therapy enhance athletic performance? The Journal of the American Dental Association, 104, 292-298.

Kaufman, RS. (1980). Case reports of TMJ repositioning to improve scoliosis and the performance by athletes. New York State Dental Journal, 46, 206-209.

Kawamori, N, Rossi, SJ, Justice, BD, Haff, EE, Pistilli, EE, O’Bryant, HS, Stone, MH, Haff, GG. (2006). Peak force and rate of force development during isometric and dynamic mid-thigh clean pulls performed at various intensities. Journal of Strength and Conditioning Research, 20(3), 483-491.

McGuigan, M. (2016). Administration, scoring, and interpretation of selected tests. In G. G. Haff \& N. T. Triplett (Eds.), Essentials of Strength Training and Conditioning (pp 259-316). Champaign, IL: Human Kinetics.

NCAA. (2011). 2011-12 NCAA Sports Medicine Handbook. Indianapolis, IN: The National Collegiate Athletic Association.

Smith, SD. (1978). Muscular strength correlated to jaw posture and the temporomandibular joint. New York State Dental Journal, 48, 278-285.

The SENIAM Project. [Online] Available: http://www.seniam.org (October 28, 2014). 